\title{
Simulation Informed CAD for 3D Nanoprinting
}

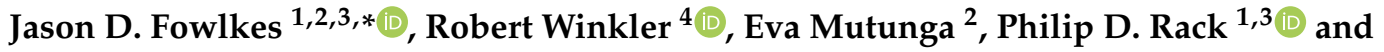 \\ Harald Plank ${ }^{4,5}$ (D) \\ 1 Center for Nanophase Materials Sciences, Oak Ridge National Laboratory, Oak Ridge, TN 37830, USA; \\ prack@utk.edu \\ 2 Bredesen Center for Interdisciplinary Research, The University of Tennessee, Knoxville, TN 37996, USA; \\ emutunga@vols.utk.edu \\ 3 Materials Science and Engineering, The University of Tennessee, Knoxville, TN 37996, USA \\ 4 Christian Doppler Laboratory for Direct-Write Fabrication of 3D Nano-Probes, Institute of Electron \\ Microscopy and Nanoanalysis, Graz University of Technology, 8010 Graz, Austria; \\ robert.winkler@felmi-zfe.at (R.W.); harald.plank@felmi-zfe.at (H.P.) \\ 5 Graz Centre for Electron Microscopy, $8010 \mathrm{Graz}$, Austria \\ * Correspondence: fowlkesjd@ornl.gov; Tel.: +865-223-2902
}

Received: 31 October 2019; Accepted: 17 December 2019; Published: 18 December 2019

\begin{abstract}
A promising 3D nanoprinting method, used to deposit nanoscale mesh style objects, is prone to non-linear distortions which limits the complexity and variety of deposit geometries. The method, focused electron beam-induced deposition (FEBID), uses a nanoscale electron probe for continuous dissociation of surface adsorbed precursor molecules which drives highly localized deposition. Three dimensional objects are deposited using a 2D digital scanning pattern-the digital beam speed controls deposition into the third, or out-of-plane dimension. Multiple computer-aided design (CAD) programs exist for FEBID mesh object definition but rely on the definition of nodes and interconnecting linear nanowires. Thus, a method is needed to prevent non-linear/bending nanowires for accurate geometric synthesis. An analytical model is derived based on simulation results, calibrated using real experiments, to ensure linear nanowire deposition to compensate for implicit beam heating that takes place during FEBID. The model subsequently compensates and informs the exposure file containing the pixel-by-pixel scanning instructions, ensuring nanowire linearity by appropriately adjusting the patterning beam speeds. The derivation of the model is presented, based on a critical mass balance revealed by simulations and the strategy used to integrate the physics-based analytical model into an existing 3D nanoprinting CAD program is overviewed.
\end{abstract}

Keywords: focused electron beam induced deposition;3D nanoprinting; Additive nanomanufacturing

\section{Introduction}

Computer simulations are commonly employed to complement nanoscale synthesis as in situ characterization is often not practical. Thus, time-dependent dynamics operative during synthesis are often unresolved and reconstruction of multiple ex situ characterization steps is necessary to try to infer time-varying progressions. Unfortunately, single experiment time-dependent behavior is only approximated using characterization results derived from multiple experiments, whether using (i) multiple process times, using the same sample, or (ii) using multiple samples with variable process times. Computer simulations can thus facilitate understanding the physical, chemical and temporal coordinates, supplementing experimental knowledge to predict deposition geometry.

A simulation strategy is presented here that moves beyond simple geometric predictions, toward the integration of compensation strategies to avoid defects for enhanced nanoscale deposition precision. Specifically, simulation results, calibrated against real experiments, are used to construct an analytical 
mathematical model for the purpose of informing computer-aided design (CAD) to prevent structural distortions during deposition. At its core, the problem is of the reaction-diffusion type and depends on multiple time-dependent physical and chemical processes. The derived analytical mathematical model presented here represents the simplest description of the nanoscale synthesis method while capturing the critical physics that governs deposition. This approach is demonstrated for a 3D nanoprinting technique.

Three dimensional nanoprinting using focused electron beam induced deposition (FEBID) [1] is one such nanoscale synthesis method with limited in situ analysis options [2,3]. In this method, a focused (nanoscale) electron probe is used to continuously dissociate precursor molecules adsorbed in the beam interaction region (BIR). In the deposition mode, a deposit accumulates over time under both continuous precursor flow and continuous beam irradiation. In the etching mode [4], the substrate is consumed.

Secondary electrons (SE) dominate deposition. SEs are created in the near surface region by the primary electron beam and the emitted SE surface flux drives precursor dissociation/fragmentation [5]. These experiments are carried out in either a conventional scanning electron microscope (SEM) or a dual electron/ion microscope equipped with a precursor injection nozzle capable of delivering molecules continuously, and at a relatively high flux, directly to the BIR. Localized precursor delivery preserves the overall high vacuum in the specimen chamber required for stable microscope operation.

Using FEBID in the 3D nanoprinting mode [6-8], mesh style objects are deposited using a network design of interconnected linear nanowires. The typical nanowire diameter spans the length scale of 25-100 nm, so in situ optical imaging techniques are not practical for FEBID. The electrical current flowing through the substrate, to ground, can be dynamically monitored during deposition $[9,10]$, which provides information of the status of deposition without influencing the deposition process. Deposit electrical properties have even been dynamically improved in situ using continuous electrical analysis coupled with machine learning [11,12]. However, information regarding the structural progress of deposition is limited. Film thickness measurements have also been carried out in situ, but this method is only appropriate for semi-infinite thin film deposition [3]. The lack of in situ characterization methods for FEBID, at least in part, motivated the creation of the 3D-FEBID simulation used here $[13,14]$.

The main purpose of the current Communication is to report an analytical mathematical model, derived from 3D-FEBID simulations that corrects for unwanted structural distortions in the final deposit. Computer simulation results are presented that (i) provide the basis for the choice of a distortion correction model, namely modulating the exposure beam speed, and (ii) the core physics used to construct the model. The initial results of the correction scheme are presented, which paves the way for a more comprehensive future Article. Specifically, the correction scheme is discussed, with an emphasis placed of the role that precursor surface concentration gradients play in causing deposit distortion, whereas heating-related effects have been revealed previously in Utke et al., Randolph et al., Mutunga et al. and Skoric et al.'s researches [15-18]: this paper leverages heavily results previously reported in Mutunga et al.'s work [17]. Further, the integration of the analytical model with the FEBID specific, CAD environment (3BiD) [19] will be the topic of a future article.

\section{Materials and Methods}

Standard nanoprinting settings for 3D-FEBID were used in the simulations, as summarized in Table 1 . The relatively high primary electron beam energy (30 keV: range: 0-30 keV) minimizes electron elastic scattering in the growing deposit, thereby maintaining spatial resolution while an appreciable secondary electron flux is emitted to induce deposition on a tractable time scale (1-10 s). The relatively low beam current setting ( $32 \mathrm{pA}$ : range: $\mathrm{pA}-\mu \mathrm{A}$ ) provides the highest spatial resolution while enabling adequate precursor replenishment in the BIR to facilitate continuous deposition. Winkler et al. [20] explains that under these favorable conditions for spatial resolution, 3D-FEBID still operates in a precursor limited reaction regime. The $\mathrm{MeCpPt}{ }^{\mathrm{IV}} \mathrm{Me}_{3}$ precursor emulated here is limited to 
monolayer surface coverage. Prior to FEBID, the initial precursor surface coverage is $0.66\left(\mathrm{C}_{\mathrm{eq}}=\right.$ 1.85 molecules $/ \mathrm{nm}^{2} \cdot \mathrm{s}$ ), which is set by both the local precursor pressure, is $\mathrm{P}=0.5 \mathrm{mTorr}$ and the substrate temperature is $\mathrm{T}_{\mathrm{o}}=294 \mathrm{~K}$.

Table 1. Simulation conditions for demonstration.

\begin{tabular}{ccc}
\hline Parameter & Definition & Value \\
\hline $\mathrm{E}_{\mathrm{o}}$ & Primary electron beam energy & $5,30 \mathrm{keV}$ \\
$\mathrm{i}_{\mathrm{b}}$ & Primary electron beam current & $25,32 \mathrm{pA}$ \\
& Primary electron beam size (FWHM) & $14.9,6 \mathrm{~nm}$ \\
& Precursor & $\mathrm{MeCpPt} \mathrm{IV}^{\mathrm{IV}}$ \\
$\tau_{\mathrm{d}}$ & Primary electron beam exposure dwell time & $\mathrm{ms}$ \\
$\Lambda$ & Exposure pixel point pitch & $1 \mathrm{~nm}$ \\
& Deposit composition & $\mathrm{PtC}_{5}$ \\
& Substrate composition & $5 \mathrm{~nm} \mathrm{SiO}_{2} / \mathrm{Si}$ \\
$\mathrm{T}_{\mathrm{o}}$ & Substrate temperature & $294 \mathrm{~K}$ \\
$\mathrm{P}$ & Precursor pressure at the substrate surface & $0.25-0.5 \mathrm{mTorr}$ \\
\hline
\end{tabular}

Simulation results are also provided for a different equilibrium precursor surface coverage of 0.50 $\left(\mathrm{C}_{\mathrm{eq}}=1.4 \mathrm{molecules} / \mathrm{nm}^{2} \cdot \mathrm{s}\right)$, a condition achieved by lowering the vapor pressure to $0.25 \mathrm{mTorr}$ at $\mathrm{T}_{\mathrm{O}}=294 \mathrm{~K}$. Lastly, controlled 3D-FEBID has been previously demonstrated over the primary electron beam energy range of 5-30 keV [20]. Thus, simulations were also executed at $E_{o}=5 \mathrm{keV}$ to span the full range of 3D nanoprinting as some initial measure of model robustness. In this case, the beam current was $25 \mathrm{pA}$, the beam size was $14.9 \mathrm{~nm}, \mathrm{P}=0.5 \mathrm{~m}$ Torr and $\mathrm{T}_{\mathrm{o}}=294 \mathrm{~K}$.

The simulation of 3D-FEBID, written and compiled in Matlab ${ }^{\circledR}$ (Version R2017b, Mathworks, Natick, MA, USA), consists of three main rate equations that collectively capture the integral physics and chemistry of 3D-FEBID.

$$
\begin{gathered}
\frac{\partial C}{\partial t}=\nabla(D(T) \nabla C)+\frac{\delta \Phi}{s_{p}}\left(s_{p}-C\right)-\frac{C}{\tau(T)}-\sigma i_{S E}^{\prime \prime} C \\
0=q_{b}^{\prime \prime \prime}+k \nabla^{2} T \\
\frac{\partial V}{\partial t}=\frac{\Omega}{s_{d}} \sigma i_{S E}^{\prime} C
\end{gathered}
$$

Table 2 defines the independent and dependent variables appearing in Equations (1)-(3).

The rate equations that govern FEBID are described with numeric references to the terms appearing on the right-hand side of each equation, moving from left to right. The surface precursor concentration (C) evolves in $(x, y, z)$ according to Equation (1) considering (i) precursor surface diffusion on the deposit, (ii) vapor phase precursor replenishment through surface adsorption, (ii) precursor surface desorption and (iv) SE-induced dissociation. Simultaneously during FEBID, the primary electron beam transfers energy to the deposit through inelastic electron energy loss, resulting in (i) Joule heating and subsequent (ii) conduction heat transfer to the substrate heat sink (Equation (2)). Finally, a physical deposit evolves in the simulation spatial domain in direct proportion to the quantity of precursor dissociated and this is described by rate Equation (3) - the FEBID deposition rate at the BIR is directly proportional to the precursor surface concentration in the BIR.

A detailed description of the simulation is provided in Fowlkes et al. and Mutunga et al.'s researches $[13,17]$. Here, only specific aspects relevant to the current work are provided. The finite difference method is used to simultaneously solve Equations (1) and (2), although Equation (2) is only updated when the primary electron beam is displaced to a new exposure pixel or if the quantity of deposition (Equation (3)) exceeds roughly one monolayer since the last temperature profile $T(x, y, z)$ update. The steady-state approximation for heat transport is possible, in part, because the heat transport time scale is 3 orders-of-magnitude faster than the monolayer deposition rate in the 
BIR [17]. Equation (3) is updated following each simulation time step to evolve the deposit volume. Lastly, the parameters $q_{b}{ }^{\prime \prime}$ and $i_{S E}$ are derived from Monte Carlo simulations of the electron-solid interaction [17]. This calculation is also updated on the order of the monolayer deposition time.

Table 2. Focused electron beam-induced deposition parameters, definitions and units. Values for these parameters are reported in Table I [17].

\begin{tabular}{ccc}
\hline Parameter & Definition & Units \\
\hline$C$ & Precursor surface concentration $(\mathrm{x}, \mathrm{y}, \mathrm{z})$ & $/ \mathrm{m}^{2}$ \\
$T$ & Temperature $(\mathrm{x}, \mathrm{y}, \mathrm{z})$ & $\mathrm{K}$ \\
$V$ & Deposit volume $(\mathrm{x}, \mathrm{y}, \mathrm{z})$ & $\mathrm{m}^{3}$ \\
$D(T)$ & Precursor surface diffusion coefficient $(\mathrm{x}, \mathrm{y}, \mathrm{z})$ via $(T)$ & $\mathrm{m}^{2} / \mathrm{s}$ \\
$\delta$ & Precursor surface sticking probability & $(0-1)$ \\
$\Phi$ & Precursor surface impingement flux & $/ \mathrm{m}^{2} \cdot \mathrm{s}$ \\
$s_{p}$ & Monolayer precursor surface coverage $\left(0 \leq C \leq s_{p}\right)$ & $/ \mathrm{m}^{2}$ \\
$\tau(T)$ & Mean precursor surface residence time $(\mathrm{x}, \mathrm{y}, \mathrm{z}) \mathrm{via}(T)$ & $\mathrm{s}$ \\
$\sigma$ & Mean, total electron impact dissociation cross-section & $\mathrm{m}^{2}$ \\
$i_{S E}$ & Emitted secondary electron current $(\mathrm{x}, \mathrm{y}, \mathrm{z})$ & $\mathrm{e}-/ \mathrm{s}$ \\
$i_{S E}{ }^{2}$ & Emitted secondary electron flux $(\mathrm{x}, \mathrm{y}, \mathrm{z})$ & $\mathrm{e}-/ \mathrm{m}^{2} \cdot \mathrm{s}$ \\
$q_{b}^{\prime \prime \prime}$ & Deposit thermal conductivity & $\mathrm{W} / \mathrm{m}^{2} \cdot \mathrm{K}$ \\
$\Omega$ & Electron beam induced heating $(\mathrm{x}, \mathrm{y}, \mathrm{z})$ & $\mathrm{W} / \mathrm{m}^{3}$ \\
$s_{d}$ & Molecular volume of deposit for $(\operatorname{PtC} 5)$ & $\mathrm{m}^{3}$ \\
$P_{\zeta}$ & Surface density of deposit $\left(\operatorname{PtC} C_{5}\right)$ & $/ \mathrm{m}^{2}$ \\
& Perimeter of pillar/segment nanowire & $\mathrm{m}$ \\
\hline
\end{tabular}

A 3D-FEBID simulation of a 'calibration structure' [13] is provided in Figure 1a. Deposition of the full range of segment angles $\zeta=0-90^{\circ}$ makes it possible to build a wide variety of mesh style objects [21]. A calibration structure consists of a vertical 'pillar' supporting a suspended 'segment'. The pillar exposure element is a vertical nanowire satisfying $\zeta=90^{\circ}$. A segment element constitutes all other possible unique exposure element angles $0 \leq \zeta<90^{\circ}$.

The pillar exposure element is deposited using a stationary primary electron beam for an extended time period $\left(\tau_{\mathrm{d}}=3.258 \mathrm{~s}\right)$. The segment element is deposited using a constant primary electron digital beam speed $\left(\mathrm{v}_{\mathrm{b}}=\Lambda / \tau_{\mathrm{d}}=1 \mathrm{~nm} / 8.19 \mathrm{~ms}=122 \mathrm{~nm} / \mathrm{s}\right)$. Segment exposure begins at the pillar axis position with deposition proceeding off the pillar tip in response to lateral primary electron beam displacement in the $(x, y)$ focal plane.

Computer-aided design carried out using the 3BiD program [19] requires multiple calibration structure deposition experiments spanning the segment angle range of $\zeta=0-90^{\circ}$ (Appendix A); the $(\zeta$ ) is measured for a set of beam exposure speeds, defined by a variable $\left(\tau_{d}\right)$ at constant $(\Lambda)$. A calibration list is generated $\left\{\zeta, \tau_{d}\right\}$. Next, the program calculates the required $(\zeta)$ for each exposure element defining the mesh style object. The value of $\left(\tau_{d}\right)$ required to deposit each exposure element is then interpolated from the $\left\{\zeta, \tau_{d}\right\}$ list. The deposition order of exposure elements is also defined using the program.

Linear deposition elements are defined in the 3BiD program and real deposition ensues under the assumption that a linear nanowire will be deposited. Returning momentarily to the calibration, $(\zeta)$ is measured at a projected length $\left(x^{\prime}=250 \mathrm{~nm}\right)$ for each segment calibration element [20]. However, the simulation presented in Figure 1a demonstrates that linearity is achieved only over a limited range, typically $(<500 \mathrm{~nm})$. Figure 1a suggests that the precursor surface concentration, which varies along the length of the segment, or $C(s)$, is a key feature leading to non-linear segment deposition because this value steadily decreases along the segment length and the deposit growth rate (Equation (3)) is directly proportional to $(C)$. Results are now presented that link the observed segment distortion with the precursor surface concentration spatial profile $C(s)$. 


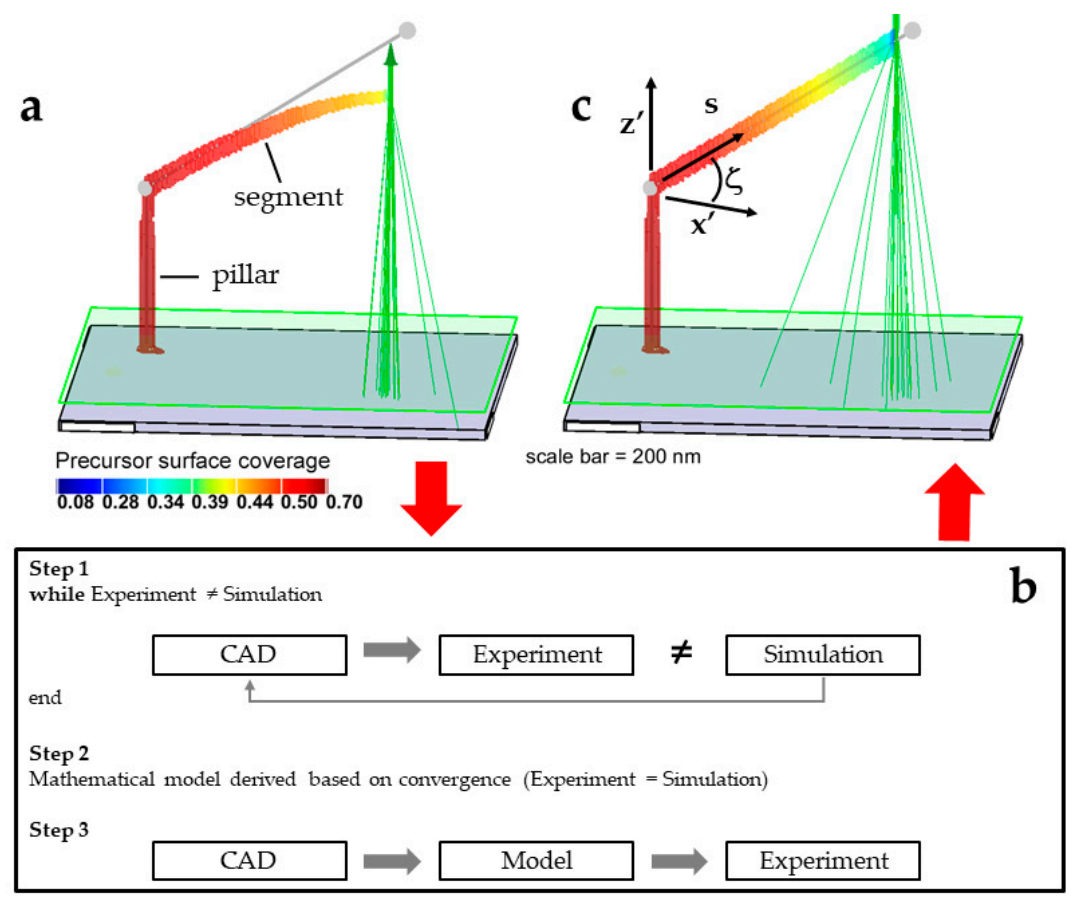

Figure 1. (a) A 3D-FEBID computer simulation of a so-called 'calibration structure' exhibiting a non-linear downward deflection/distortion causing the experimental final deposit to deviate from the CAD design (grey sphere-stick model). The precursor surface concentration is color-coded and presented as a fraction of monolayer coverage. The distance measured from the substrate along the deposit is given by $(\mathrm{s})$, the total deposit length is $\left(\mathrm{S}_{\mathrm{T}}\right)$; $(\mathbf{b})$ The proposed distortion correction scheme is based on (Step 1) a computer simulation that mimics experiments. In Step 2, the most important physics governing deposition is revealed which is incorporated into an analytical model. Ideally, the analytical mathematical model, called the 'dwell time compensation method', can be derived based on physical/chemical principles to ultimately correct for geometric distortions; (c) 3D-FEBID of the calibration structure following implementation of the analytical correction model described conceptually in the Discussion section.

\section{Results}

The deposition of a typical calibration structure was simulated to predict the precursor surface concentration profile $C(s)$ along the $3 \mathrm{D}$ deposit at various values of total deposit length $\left(\mathrm{S}_{\mathrm{T}}\right)$. These data are presented in Figure 2a for a total deposit length of $S_{\mathrm{T}} \cong 650$ (red), 890 (yellow), 1120 (green) and $1300 \mathrm{~nm}$ (blue), see solid lines. Importantly, the solid lines represent deposition without distortion compensation, see Figure 1a. The presentation of the results focuses on the segment deposition, which takes place after pillar deposition, i.e., $\mathrm{S}_{\mathrm{T}}>500 \mathrm{~nm}$. The complementary temperature profile for each case is shown in Figure 2b. $(C)$ and $(T)$ represent averages derived from data acquisition nodes $(x, y, z)$ spaced evenly along the centerline of the deposit. Only surface deposit voxels contribute to the average and to contribute to this average, they must lie within a spherical volume, centered on each node, with a characteristic radius of $40 \mathrm{~nm}$. Moreover, each $C(s)$ profile, shown in Figure 2a, was derived at the end of the pixel dwell time, just prior to the beam displacement to the next exposure pixel. Lastly, in the results analysis presented below, $C(s)$ will be used to refer to any variation in $(C)$ or $(T)$ along the segment, at a fixed segment length $\left(\mathrm{S}_{\mathrm{T}}\right)$, e.g., each line profile shown in Figure 2. At other times, it is useful to discuss how the precursor surface concentration at the segment tip, or BIR, varies with total segment length, i.e., $C\left(\mathrm{~S}_{\mathrm{T}}\right)$. For example, the four circular data points shown in Figure 2 represent the $C\left(\mathrm{~S}_{\mathrm{T}}\right)$ trend. 


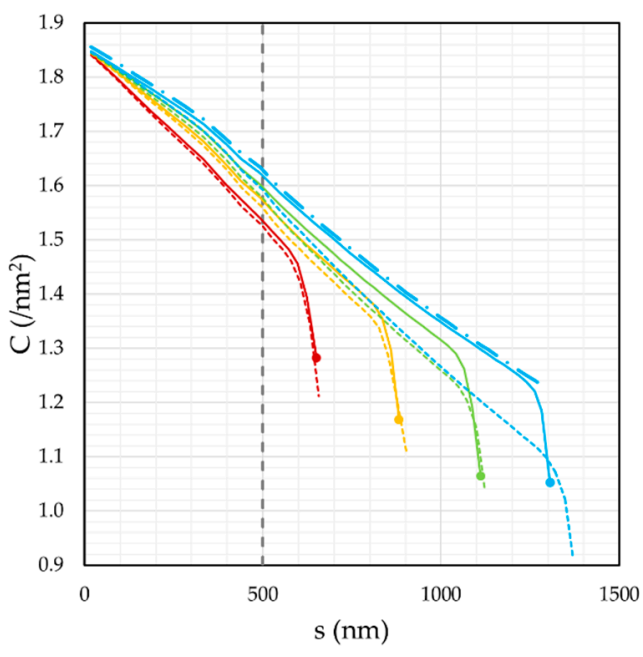

(a)

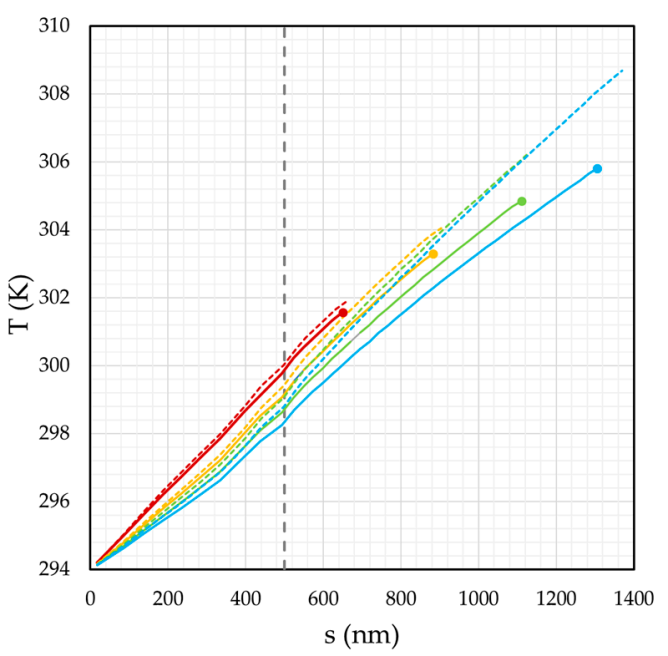

(b)

Figure 2. (a) The precursor surface concentration $(C)$ along the deposit axis $(s)$ at four different stages of deposition. The solid lines represent deposition without segment deflection compensation while the dashed lines represent deposition with compensation. Results are shown for a total deposit length $\left(\mathrm{S}_{\mathrm{T}}\right)$ $\cong 650$ (red), 890 (yellow), 1120 (green) and $1300 \mathrm{~nm}$ (blue). The hatched grey line shows the boundary between the pillar (s $<500 \mathrm{~nm}$ ) and segment (s > $500 \mathrm{~nm}$ ) exposure elements.; (b) The complementary surface temperature $(T)$ profile for the four stages of deposition shown in panel (a).

\subsection{Precursor Surface Concentration Outside the Beam-impact Region}

To begin, we will examine the surface concentration and temperature for a total deposit length of $\mathrm{S}_{\mathrm{T}} \cong 1300 \mathrm{~nm}(-) . C(s)$ steadily decreases, with increasing $s$, over the range $\mathrm{s}=0-1200 \mathrm{~nm}$. This trend is directly caused by the thermal gradient directed along the deposit length (Figure $2 b,-)$. Joule heating in the BIR is induced by the inelastic scattering of the internally scattered electrons as they pass through the deposit (Figure 1a). The magnitude of Joule heating scales with (i) the z-thickness of the segment in the BIR which controls the quantity of energy absorbed during beam transmission, (ii) the total length of the deposit which increases thermal resistance and promotes heating, and (iii) inversely with the cross-sectional area $(A)$ of the segment as the thermal resistance is inversely proportional to this area [17]. Thermal resistance $\left(R_{T}\right)$ increases due to points 2 and 3, i.e., $R_{T}=\mathrm{S}_{\mathrm{T}} /(\mathrm{k} \times A)$. The exact role that beam heating plays is revealed by way of the complementary $C(s)$ profile (o-). This hypothetical $C(s)$ profile represents the equilibrium precursor surface concentration $\left(C_{e q}\right)$ that would be present along the segment if it were possible to heat the segment with the beam at the segment tip without consuming the precursor. This is implemented by calculating $\left(C_{e q}\right)$ solely based on physisorption (terms 3 and 4 in Equation (2)) and $T(s)$ (Appendix B). In other words, this value represents the maximum possible value of $(C)$, at any position (s), given the current surface temperature of $(T)$. The simulated profile $C(s)$ nearly equals $C_{e q}(s)$ over the range $\mathrm{s}=0-1200 \mathrm{~nm}$ indicating that temperature-dependent vapor phase replenishment/desorption determines $C(s)$ outside the BIR. The limitations of physical adsorption model are briefly described in Appendix B.

\subsection{Precursor Surface Concentration Inside the Beam-Impact Region}

A drastic reduction in precursor surface concentration occurs in the BIR (s $>1200 \mathrm{~nm}$, Figure 2a, -) over the distance of $\Delta \mathrm{s}=80 \mathrm{~nm}$ measured from the end of the segment. The mechanism for the formation of this concentration gradient $(\mathrm{d} C / \mathrm{d} s)$ has been reported previously (Figure 10, in [17])-SE induced precursor dissociation (term 4, Equation (1)) generates a precursor gradient at the BIR boundary that maintains precursor flow to the BIR by surface diffusion (term 1, Equation (1)). Unfortunately, the relatively large $\mathrm{MeCpPt}^{\mathrm{IV}} \mathrm{Me}_{3}$ organometallic molecule has a small surface diffusion coefficient. 
How, then, does precursor surface diffusion sustain deposition? Precursor surface diffusion is sustained by the large concentration gradient self-imposed by beam dissociation in the BIR, i.e., precursor flux $D \times \mathrm{d} C / \mathrm{d}$ s. The compensation strategy proposed later hinges on the stability of this physical process. The stability is now quantified using a normalization procedure, as outlined below.

\subsection{Segment Deposition-A Stable Physical Growth Mechanism}

Multiple precursor surface concentration profiles $C(s)$ are shown in Figure $2 \mathrm{a}$ as a function of the total deposit length $\left(\mathrm{S}_{\mathrm{T}}\right)$. The general function shape of $C(s)$ is approximately constant, independent of $\mathrm{S}_{\mathrm{T}}$. This is clearly revealed by normalization (Figure $\mathrm{A} 1$ ) of $C(s)$ for each $\mathrm{S}_{\mathrm{T}}$. First, the normalization $C / C_{e q}$ reveals that physical absorption controls precursor replenishment along the segment element surface, except at the segment tip region, i.e., $C / C_{e q} \approx 1$. Secondly, the relatively steep precursor gradient, observed at the BIR, is found to be independent of $\left(\mathrm{S}_{\mathrm{T}}\right)$. This is clearly revealed when the origin for $s$ is shifted to the total deposit length $\left(\mathrm{S}_{\mathrm{T}}\right)$ (Figure A1). The normalization analysis indicates that the physical mechanism driving 3D deposition is stable during segment growth, over a critical range of useful 3D nanoprinting $\left(\mathrm{S}_{\mathrm{T}}<1000 \mathrm{~nm}\right)$. The important question now arises, "If the physical mechanism driving deposition is stable, what then causes the non-linear distortion?". The answer is: the increase in the precursor surface desorption flux inside, and outside, the BIR which occurs in response to the steady temperature rise at the segment tip as $\left(S_{T}\right)$ increases (Figure $2 b, 0 \rightarrow 0$ ). This causes a complementary reduction in $C\left(\mathrm{~S}_{\mathrm{T}}\right)($ Figure $2 \mathrm{a}, \bullet \rightarrow \bullet)$ and the final geometry exhibits a non-linear segment deflection (Figure 1a).

\section{Discussion}

\subsection{A Compensation Model for the Case of Non-linear Segment Deposition}

The 3D-FEBID simulation reveals that the variation of the precursor surface concentration in the beam impact region $C\left(S_{T}\right)$, due to $\tau(T)$, plays a pivotal role in determining segment linearity. The deflection compensation strategy aims to offset the continuous decrease in $C\left(S_{T}\right)$ using beam patterning deceleration. Specifically, a continuous beam deceleration is proposed, where the pixel point pitch $(\Lambda)$ remains fixed, while the primary electron beam dwell time per pixel $\left(\tau_{d}\right)$ is appropriately increased. Recently, a high-quality helix geometry was deposited using FEBID by experimentally tailoring beam deceleration [22]. Thus, the performance of the strategy relies on the quality of the prediction for $\left(\tau_{d}\right)$ as a function of $\left(\mathrm{S}_{\mathrm{T}}\right)$.

Derivation of the mathematical model begins with the vertical growth rate in the segment reference frame (Figure $\left.1 c,\left\{\mathrm{x}^{\prime}, \mathrm{z}^{\prime}\right\}\right)$. The FEBID vertical deposition rate $\left(\mathrm{d} z^{\prime} / d t\right)$ during linear segment deposition is defined as

$$
\frac{d z^{\prime}}{d t}=\Omega \sigma i_{S E}^{\prime \prime} C
$$

In the ideal linear approximation of 3D-FEBID, $C$ is not a function of segment length and consequently, not time dependent (Appendix D). Unfortunately, this is not the case for the simulations reported here and a spatial dependence must be introduced. First, a chain rule expansion introduces beam deceleration into the vertical growth rate expression;

$$
\frac{d z^{\prime}}{d x^{\prime}} \frac{d x^{\prime}}{d t}=\Omega \sigma i_{S E}^{\prime \prime} C\left(x^{\prime}\right)
$$

Only then is the constraint of constant precursor surface concentration relaxed to $C\left(x^{\prime}\right)$. It is more convenient to execute the expansion in $\left(x^{\prime}\right)$, instead of $\left(\mathrm{S}_{\mathrm{T}}\right)$, because the beam patterning takes place in $\left(x^{\prime}\right)$; primary electron beam scanning deceleration in the focal plane is represented by $\left(\mathrm{d} x^{\prime} / \mathrm{d} t\right)$ which correctly traces the projected length of the segment in this plane as a function of time.

The next mathematical step is to specify the desired segment angle. The term $\left(\mathrm{d} z^{\prime} / \mathrm{d} x^{\prime}\right)$ is used to introduce $(\zeta)$; if a linear deposition mode is desired, then this value should remain constant during 
segment deposition. These facts validate the use of $\tan \zeta=\mathrm{d} z^{\prime} / \mathrm{d} x^{\prime}$. Equation (5) is now cast in a more useful form by (i) imposing the design, or CAD specification for ( $\zeta$ ), (ii) separating constant parameters (left-hand side) from variable parameters (right-hand side) and (iii) converting from beam deceleration $\left(\mathrm{d} x^{\prime} / \mathrm{d} t^{\prime}\right)$ to electron dose per unit length $\left(\mathrm{d} t^{\prime} / \mathrm{d} x^{\prime}\right)$ :

$$
\frac{d t}{d x^{\prime}} C\left(x^{\prime}\right)=\frac{\tan \zeta}{\Omega \sigma i_{S E}^{\prime \prime}}
$$

Thus, with a proper description of the decrease in $C\left(x^{\prime}\right)$, one can easily compute the required increase in the quantity $\left(\mathrm{d} t / \mathrm{d} x^{\prime}\right)$ to ensure that the right-hand side remains constant during segment deposition (Appendix E). Fortunately, the simulation results presented above in concert with results reported in Mutunga et al.'s research [17], identify a pathway to determine $C\left(x^{\prime}\right)$.

\subsection{The Definitive Surface Mass Balance Dictating 3D-FEBID}

The 3D-FEBID simulation reveals a surface mass, i.e., precursor, flow rate boundary condition that primarily dictates the nanoprinting mode deposition physics. This boundary condition provides a means to determine the BIR precursor surface concentration as a function of projected segment length $C\left(x^{\prime}\right)$ (Appendix F). Specifically, segment deposition is strongly controlled by the balance between the precursor surface diffusion rate and the precursor dissociation rate at the BIR boundary, or;

$$
D \frac{d C}{d s} P_{\zeta}=\sigma i_{S E} C
$$

where the units are molecules/s. Linearization of the boundary condition makes it possible to cast the equation in terms of known quantities, derived from simulation

$$
D \frac{\mathrm{C}_{\mathrm{eq}}\left(T\left(x_{b}^{\prime}\right)\right)-\mathrm{C}_{\mathrm{BIR}}}{\Delta s}=\sigma i_{S E} \mathrm{C}_{\mathrm{BIR}}
$$

where $x_{b}^{\prime}$ is the position of the BIR boundary. Several assumptions were made in constructing this simplification, which are based on the simulation results presented in the Results section. The $\mathrm{d} C / \mathrm{d} s$ region was measured from Figure 2a as $\Delta s=80 \mathrm{~nm}$, which is stable as a function of segment length (Figure A1). The BIR boundary concentration is taken as the equilibrium concentration $\left(C_{e q}\right)$, determined by the balance between adsorption and desorption, since the agreement between $C(s)$ and $C_{e q}(s)$ extends nearly to the BIR boundary during segment deposition (Figure 2a, - vs -).

\subsection{The Dwell Time Compensation Model for Linear 3D Nanoprinting by FEBID}

The analytical dwell time compensation model, so-called because the dwell time is the variable parameter in $\left(x^{\prime}\right)$, may now be constructed by combining Equations (6) and (8) and Appendix B;

$$
\frac{d t}{d x^{\prime}}\left(x^{\prime}\right)=\frac{\frac{\tan \zeta}{\sigma \Omega i_{S E^{\prime \prime}}}\left(1+\frac{\sigma i_{S E} \Delta s}{P_{\zeta} D(T)}\right)}{\frac{\delta \Phi}{\frac{\delta \Phi}{s_{d}}+\frac{1}{\tau(T)}}}
$$

A complementary temperature prediction is required as a function of segment length;

$$
T\left(x^{\prime}\right)=T(0)+\frac{d T}{d x^{\prime}} x^{\prime}
$$

A thermal circuit network model was integrated with the 3BiD program, based in part on the 1D thermal modelling reported in Mutunga et al.'s research [17]. The network model is an automated prediction and will be the topic of a future article. Please note that dwell time compensation has only been explored for a carbon-rich nanoparticle-matrix composite, e.g., $\mathrm{PtC}_{5}$, in the limit where 
the amorphous carbon phase dominates heat transport [17]. In fact, it is possible that metal-rich deposits will not require exposure correction for heating effects because the relatively larger thermal conductivity of metals.

Digital beam scanning is used during real 3D-FEBID experiments, whereas the analog function was derived for compensation (Equation (9)). The digital form can be derived from the analog model for any given electron beam position as

$$
\tau_{d}(n \cdot \Lambda)=\int_{(n-1) \cdot \Lambda}^{n \cdot \Lambda} \frac{d t}{d x^{\prime}} d x^{\prime}
$$

The variable $n$ is an integer and defines the beam displacement in integer multiples of the pixel point pitch $(\Lambda) . n$ begins at the initial value of $n=n_{0}$, where $n_{0}>>1$ which delays the compensation to allow for segment nucleation, and a final value of $n=N$ which is the total number of scanning pixels required to deposit the complete segment exposure element.

The 3D-FEBID simulation with the applied correction is presented in Figure 1c. The complementary $C(s)$ and $T(s)$ profiles are presented in Figure 2a,b, respectively. For clarity, Figure 3a,b show 2D slices through the simulation domain for the calibration structure images presented in Figure $1 \mathrm{a}, \mathrm{c}$.

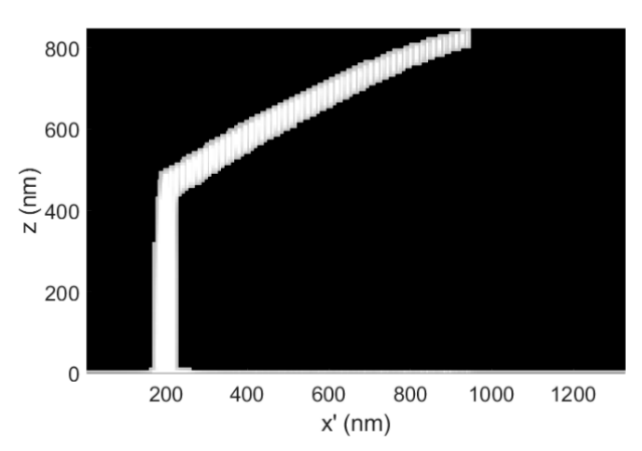

(a)

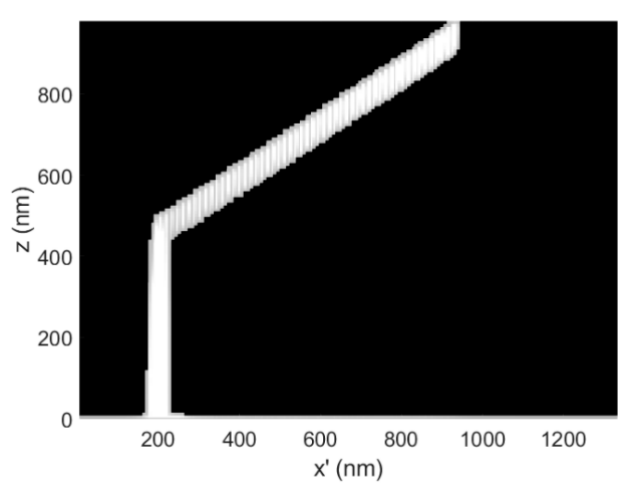

(b)

Figure 3. (a) 3D-FEBID simulation calibration structure cross-section for the uncorrected, as-deposited non-linear case. The dwell time per pixel $\tau_{\mathrm{d}}=8.19 \mathrm{~ms}$ for segment deposition, $\mathrm{E}_{\mathrm{o}}=30 \mathrm{keV}, \mathrm{i}_{\mathrm{b}}=32 \mathrm{pA}$ and $\mathrm{P}=0.5$ mTorr; (b) The distortion corrected, complementary 3D-FEBID simulation cross-section demonstrating the deposition of the intended linear segment. In this case, the dwell time per pixel spanned the range $\tau_{d}=8.19-12.23 \mathrm{~ms}$. CAD specified a pillar length of $500 \mathrm{~nm}$, a segment length of $1000 \mathrm{~nm}$ and a segment angle of $\zeta=30^{\circ}$.

The dwell time compensation method works by steadily increasing the deposition time per pixel to compensate for the steady decline in the precursor surface concentration in the BIR as the segment length increases. Ultimately, this yields a constant vertical growth rate per pixel and linear growth. As expected, the compensation tactic is accompanied by a temperature increase, for any given segment length (see Figure 2b, - for all colors) because the z-thickness of the deposit is preserved along the segment length (Figure 3b). Conversely, during uncorrected deposition, the thickness steadily decreases (Figure 3a) due to the steadily decreasing growth rate, reducing the magnitude of Joule heating (Figure 3a)-Joule heating scales proportionally with z-thickness in the BIR.

A refresh time tactic is an enticing alternative to the dwell time compensation method where the beam is periodically displaced away from the deposit to allow for periodic cooling. Unfortunately, beam heating is implicit and cannot be avoided practically because the time scale to achieve steady-state heat transfer is on the order of $10^{-7} \mathrm{~s}$ while the total deposition and refresh times per pixel are on the order of $10^{-3} \mathrm{~s}$ for 3D-FEBID. Thus, the beam would have to be blanked on the order of $10^{4}$ times per exposure pixel to deposit only at a low temperature. This exposure frequency approaches the 
instrumentation limit. Furthermore, deposition under these conditions would be time-dependent in precursor surface concentration $(\mathrm{d} C / \mathrm{d} t)$ because the beam cooling/heating rate is dynamic at this frequency - operation under steady-state conditions is more predictable. Please note that it is not our intention to discredit a refresh time-based approach but rather to highlight the advantage of operating under steady-state conditions. Thus, although the dwell time compensation operates at a slightly higher deposition temperature during growth, steady-state deposition affords predictability.

Interestingly, the compensation equation anticipates the temperature-rise accurately enough to impose the correct linear compensation (Figure 3b) even though the temperatures reached during the corrected simulation exceed those achieved during uncorrected deposition. This is probably due to the stability of the precursor flow balance at the BIR boundary (Figure A1) that ultimately minimizes the segment non-linearity, making compensation possible. Thus, pixel dwell time compensation appears to work, not only in an interpolated way, but also seems to extrapolate correctly. Only a comprehensive experimental examination can confirm this speculation.

The dwell time compensation method was also demonstrated at a lower precursor surface concentration, where the precursor vapor pressure was reduced from $\mathrm{P}=0.5 \mathrm{~m}$ Torr to $\mathrm{P}=0.25 \mathrm{mTorr}$ during deposition (Figure 4). Ultimately, the decrease in pressure translates into a general decrease in $C$ at all points $s$, including the BIR. Figure $4 \mathrm{~b}$ shows that the compensation method successfully drives linear segment deposition as expected-even though $C_{\text {BIR }}$ does not explicitly appear in Equation (9), the term appears as a variable in the derivation.

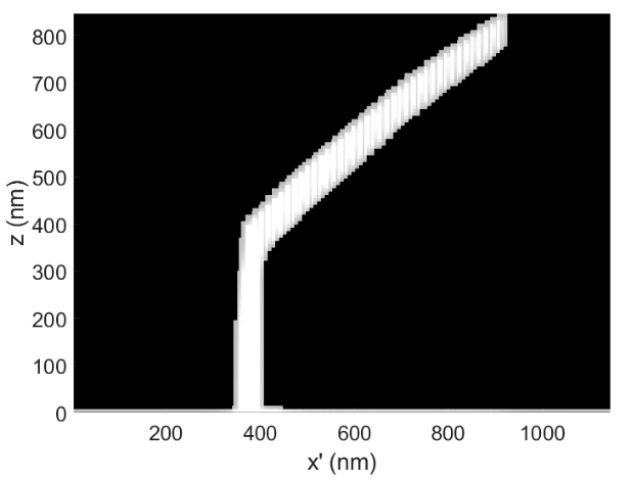

(a)

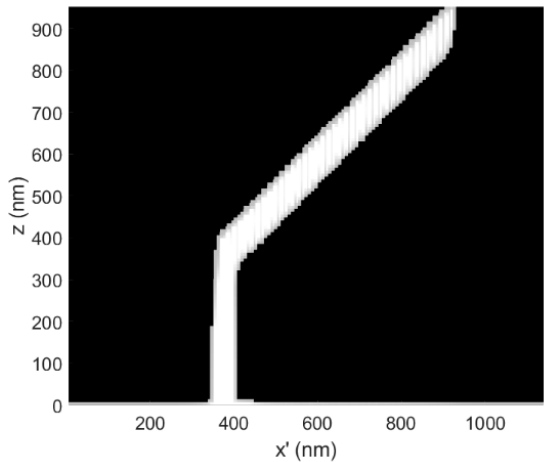

(b)

Figure 4. (a) A 3D-FEBID simulation demonstrating the uncorrected deposition of a calibration structure with a CAD specified segment angle of $\zeta=54^{\circ}$, a pillar length of $400 \mathrm{~nm}$, a segment length of $800 \mathrm{~nm}$. The dwell time per pixel for segment deposition was $\tau_{\mathrm{d}}=15 \mathrm{~ms}, \mathrm{E}_{\mathrm{o}}=30 \mathrm{keV}, \mathrm{i}_{\mathrm{b}}=32 \mathrm{pA}$ and $\mathrm{P}=0.25$ mTorr; (b) The complementary 3D-FEBID simulation for the corrected situation. The dwell time per pixel spanned the range $\tau_{d}=15-26.86 \mathrm{~ms}$.

The dwell time compensation method was also tested at the lower end of the 3D-FEBID primary electron beam energy range $\left(E_{O}=5 \mathrm{keV}\right)$ to explore the utility of compensation over the available nanoprinting range specified in the Methods section (Figure 5). GIS conditions were held constant relative to the simulation results reported in Figure 3 for $E_{o}=30 \mathrm{keV}$ for comparison purposes. The electron-solid interaction volume is strongly confined to the BIR at $E_{o}=5 \mathrm{keV}$, whereas at $\mathrm{E}_{\mathrm{O}}=30 \mathrm{keV}$, the beam is only weakly scattered in the BIR. As shown below, the segment element (Figure 5a) gently curves downward for the uncorrected case (see $x^{\prime}>600 \mathrm{~nm}$ ) while the dwell time compensation method (Figure $5 b$ ) is successful at producing a linear segment element.

The deposition simulation shown in Figure 5 was extended to a multi-level beam exposure to investigate the compatibility of the method with a nanowire 'kink', as well as a segment exceeding $1 \mu \mathrm{m}$ in length. The kink was introduced in the CAD by exposing a second segment with an orientation of $\zeta=54^{\circ}$ and a length of $800 \mathrm{~nm}$ but with an in-plane/substrate rotation of $\pi$ relative to the underlying supporting segment. Such a kink is a common feature in mesh object 3D models. 


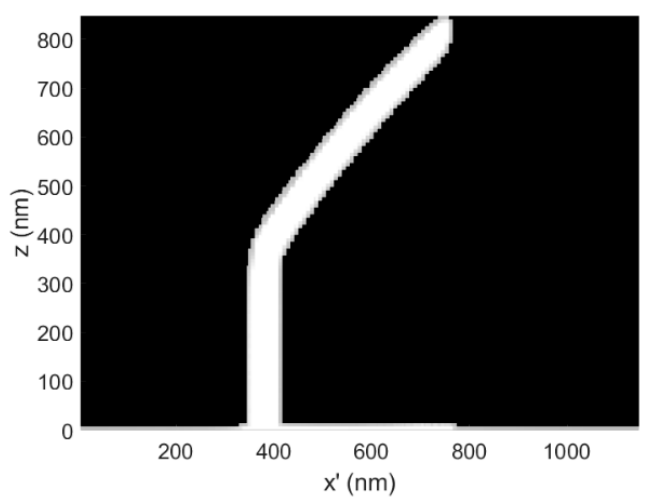

(a)

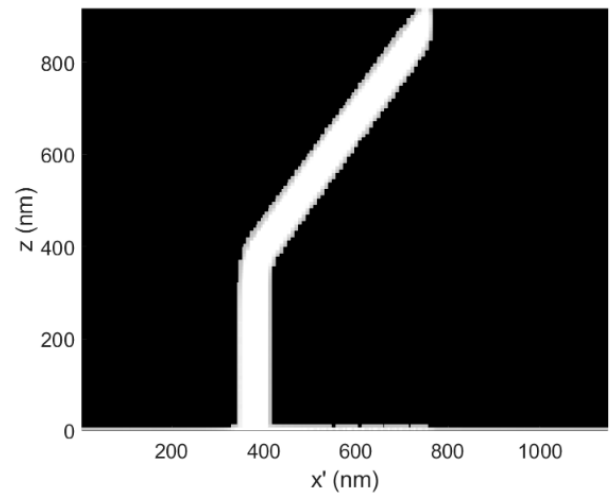

(b)

Figure 5. (a) A 3D-FEBID simulation of a calibration structure deposited at $\mathrm{E}_{\mathrm{o}}=5 \mathrm{keV}$ and $\mathrm{i}_{\mathrm{b}}=25 \mathrm{pA}$. The CAD specified segment angle was $\zeta=54^{\circ}$, the pillar element is $400 \mathrm{~nm}$ long and the segment element is $800 \mathrm{~nm}$ in length. The dwell time per pixel for the segment element was $\tau_{d}=15 \mathrm{~ms}$; (b) The distortion corrected, complementary 3D-FEBID simulation cross-section where the dwell time per pixel spanned the range $\tau_{d}=15-21.94 \mathrm{~ms}$ during segment deposition.

The dwell time compensation method was also successful at producing a complete set of linear, interconnected nanowires for this more complex geometry, suggesting that the model may be compatible with the kink feature (Figure 6b). For example, the superimposed arrow aligned with the initial segment is oriented at $\zeta=54^{\circ}$. The measurement arrow was then flipped in the horizontal image dimension and moved to the second segment to show the change in segment angle for the second segment, relative to the first segment. A change in segment angle of $\Delta \zeta=-2^{\circ}$ was observed for the second segment to $\zeta=52^{\circ}$. This example is intended to estimate the potential accumulation of segment angle errors as additional 3D nanoprinting exposure levels are added to a 3D mesh style object model.

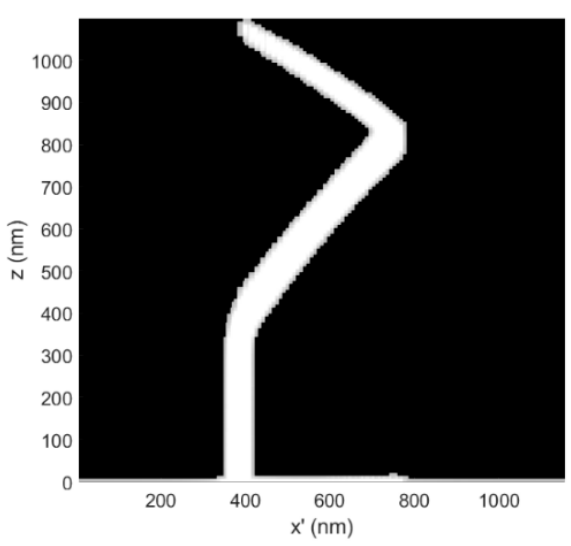

(a)

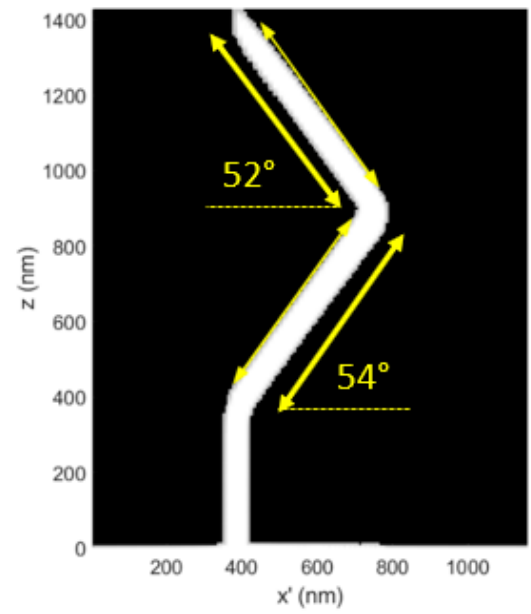

(b)

Figure 6. (a) A 3D-FEBID simulation of a multi-level deposit exposure without dwell time compensation using $\mathrm{E}_{\mathrm{O}}=5 \mathrm{keV}, \mathrm{i}_{\mathrm{b}}=25 \mathrm{pA}, \mathrm{P}=0.5 \mathrm{mTorr}$ and $\mathrm{T}_{\mathrm{o}}=294 \mathrm{~K}$. The initial segment angle at take-off is $\zeta=54^{\circ}$ and the segment element length is $800 \mathrm{~nm}$. A second segment element was deposited with an in-plane/substrate plane rotation of $(\pi)$ to simultaneously simulate (i) a doubling of segment length and (ii) the incorporation of a ' $\mathrm{kink}^{\prime}$ in the 3D geometry. Thus, the segment angle specified in the CAD file for the second segment is also $\zeta=54^{\circ}$. (b) Dwell time compensation results in the desired linearization of the exposure elements. The dwell time range calculated for the first segment was $\tau_{d}=15-21.94 \mathrm{~ms}$. The second segment required the dwell time range of $\tau_{d}=15-41.26 \mathrm{~ms}$. 
The multi-layer exposure simulation demonstration suggests that the dwell time compensation method may be extended to (i) more complex segment interconnections and (ii) exposure elements exceeding $1 \mu \mathrm{m}$. Only future experiments will confirm these simulated predictions.

\section{Conclusions}

The dwell time compensation method, strategically presented in Figure 1b, is now summarized. The 3D-FEBID simulation was used to pinpoint the most relevant physics which controls segment deposition during 3D-FEBID. The 3D-FEBID simulation revealed that Joule heating, caused by the transmitted primary electron beam, induces a temperature-dependent precursor surface desorption flux gradient along the segment. Ultimately, this gradient induces a steady reduction in the precursor surface concentration in the beam impact region (BIR) as a function of segment length. Fortunately, the simulation also reveals that the precursor surface flow balance between surface diffusion and precursor dissociation is stable during segment growth. This stability at least minimizes the impact of the precursor surface concentration in the BIR, which is realized in the final segment geometry as a relatively slight non-linear deflection of the segment when a linear deposit is desired.

Revelation of the 3D deposition mechanism made it possible to derive a simple analytical description of 3D-FEBID for direct application in the CAD phase of 3D-FEBID. Key parameters derived from the simulation, such as (i) the characteristic length of the precursor surface concentration gradient $(\Delta s)$ feeding deposition in the BIR, (ii) the emitted secondary electron surface current and flux ( $i_{S E}$ and $\left.i_{S E}{ }^{\prime \prime}\right)$, and (iii) the segment temperature $T\left(x^{\prime}\right)$, are directly applied to the analytical model. This correction should, in principle, be valid for future deposition experiments at the primary electron acceleration energy and beam current settings for which they were derived. In the future, the dwell time compensation method will be integrated with the FEBID CAD 3BiD program for general use. The quality of the dwell time correction method is currently under study experimentally.

Author Contributions: Conceptualization, J.D.F. and H.P.; interpretation of results, E.M., P.D.R., R.W. and H.P.; simulations, J.D.F. and E.M.; writing—original draft preparation, J.D.F.; writing—review and editing, all; visualization, J.D.F., E.M. All authors have read and agreed to the published version of the manuscript.

Funding: HP and RW acknowledge the financial support by the Christian Doppler Research Association (CDL-DEFINE), Austrian Cooperative Research (ACR), FFG Beyond Europe project (AIM, No. 11056459). The financial support by the Austrian Federal Ministry for Digital and Economic Affairs and the National Foundation for Research, Technology and Development is gratefully acknowledged. J.D.F. and E.M. acknowledge support from the U.S. Department of Energy (DOE) under grant No\# KC0403040 ERKCZ01.

Acknowledgments: Harald Plank and Robert Winkler thank Ferdinand Hofer for scientific discussions and for financial support concerning instrumentation. All the authors acknowledge that the computer simulations and theoretical development were conducted at the Center for Nanophase Materials Sciences, which is a DOE Office of Science User Facility.

Conflicts of Interest: The authors declare no conflict of interest.

\section{Appendix A}

In general, a set of calibration structures must be grown at a constant value of (i) beam acceleration energy ( $E_{o}$ ), (ii) beam current ( $\mathrm{i}_{\mathrm{b}}$ ), (iii) beam size/focus and (iv) a fixed gas-injection system (GIS) nozzle position. If any of these variables change, a new calibration structure experimental set is recommended $[8,19,20]$.

\section{Appendix B}

The equilibrium, monolayer precursor surface concentration determined by temperature-dependent physisorption is

$$
C_{e q}(s)=\frac{\delta \Phi}{\frac{\delta \Phi}{s_{p}}+\frac{1}{k_{o}} e^{\frac{E_{a}}{k_{B} T(s)}}}
$$


where $\delta$ is the sticking probability of incident precursor molecules from the vapor phase, $\Phi$ is the vapor phase precursor impingement flux, $k_{o}$ is the precursor surface desorption attempt frequency, $E_{a}$ is the activation energy for physisorption, $k_{B}$ is Boltzmann's constant, and $T(s)$ is the surface temperature at the position along the calibration structure $(s)$.

This model of physical adsorption/desorption is valid for all fractional coverages, up to a single monolayer of precursor coverage, or

$$
\frac{C}{s_{d}}<1
$$

For example, fractional, monolayer precursor surface coverages occur during FEBID using multiple precursors including $\mathrm{W}\left(\mathrm{CO}_{6}\right), \mathrm{Me}_{2} \mathrm{Au}^{\mathrm{III}}(\mathrm{acac})$, and $\mathrm{MeCpPt}^{\mathrm{IV}} \mathrm{Me}_{3}$. The dwell time compensation model reported here can be extended to other models of surface coverage by simply replacing the equilibrium coverage equation $C_{e q}$ to the appropriate model, e.g., see Sanz-Hernández et al.'s research [23].

\section{Appendix C}

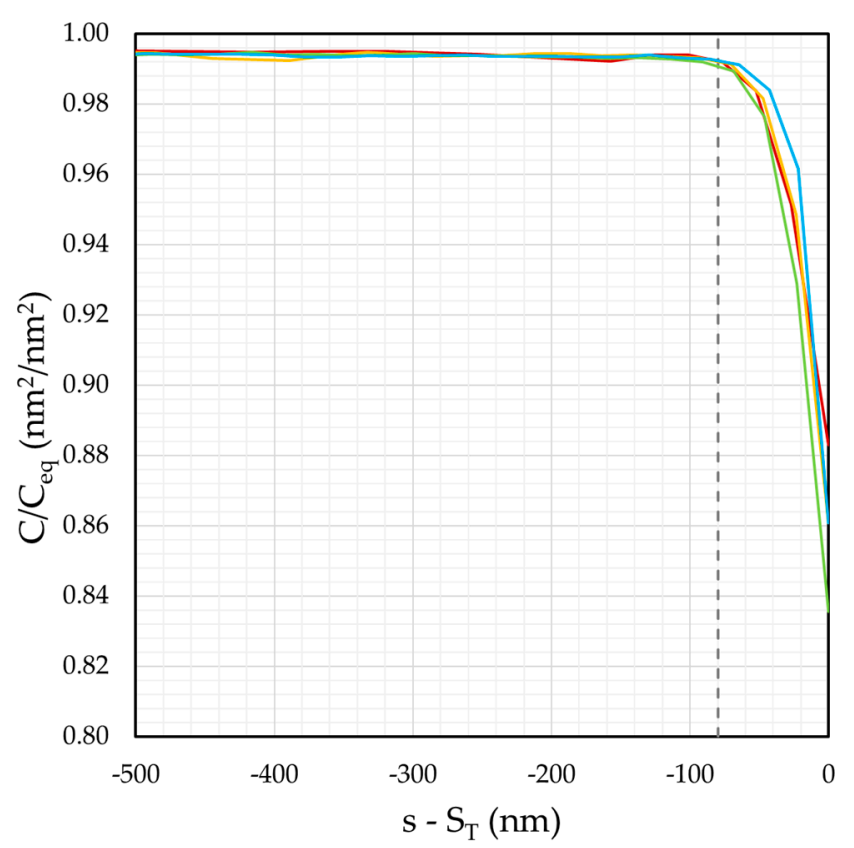

Figure A1. (a) The normalized precursor surface concentration $\left(C / C_{e q}\right)$ profile along the calibration element centerline (s) for each profile previously shown in Figure 2a. In addition, only simulations without distortion correction are shown (Figure 2a, - for all colors). The concentration is normalized to the equilibrium concentration $\left(C_{e q}\right)$ (Appendix B). The position origin has been shifted to the total deposit length $\left(\mathrm{s}-\mathrm{S}_{\mathrm{T}}\right)$ for each normalized concentration profile. The hatched line indicates that the characteristic region defining the relatively steep concentration gradient $(\Delta s=80 \mathrm{~nm})$, feeding precursor to the BIR, is nearly constant, regardless of the total calibration structure length $\left(\mathrm{S}_{\mathrm{T}}\right)$. The total deposit length for each case is $S_{\mathrm{T}} \cong 650$ (red), 890 (yellow), 1120 (green) and $1300 \mathrm{~nm}$ (blue).

\section{Appendix D}

Time $(\mathrm{t})$ and space $\left(\mathrm{S}_{\mathrm{T}}\right)$ are interchangeable considering that $S_{T}$ evolves linearly with $t$ due to the nature of FEBID where deposition occurs in the form of continuous directed growth where new deposit forms the 'substrate' for future deposition. The beam exposure speed implicitly relates the two dimensions because FEBID relies on continuous beam/tip overlap to maintain continuous growth. In other words, natural feedback maintains deposition as opposed to, e.g., an artificial feedback loop present during processing. 


\section{Appendix E}

Although the secondary electron current density $\left(i_{S E}{ }^{\prime \prime}\right)$ does not vary with the total segment length $\left(\mathrm{S}_{\mathrm{T}}\right)$, it does vary with the segment angle $(\zeta)$. This variation will be provided in a future article and the variation is included in the current version of the dwell compensation program.

\section{Appendix F}

A subtle but important note: 'boundary condition', as used here, does not refer to a boundary condition applied in the 3D-FEBID simulation but rather to a boundary condition that plays a key role in affecting the 3D-FEBID deposition process.

\section{References}

1. Utke, I.; Hoffmann, P.; Melngailis, J. Gas-assisted focused electron beam and ion beam processing and fabrication. J. Vac. Sci. Technol. B 2008, 26, 1197-1276. [CrossRef]

2. Bret, T.; Utke, I.; Gaillard, C.; Hoffmann, P. Periodic structure formation by focused electron-beam-induced deposition. J. Vac. Sci. Technol. B 2004, 22, 2504-2510. [CrossRef]

3. Perentes, A.; Bret, T.; Utke, I.; Hoffmann, P.; Vaupel, M. Real-time reflectometry-controlled focused-electron-beam-induced deposition of transparent materials. J. Vac. Sci. Technol. B 2006, 24, 587-591. [CrossRef]

4. Toth, M.; Lobo, C.; Friedli, V.; Szkudlarek, A.; Utke, I. Continuum models of focused electron beam induced processing. Beilstein. J. Nanotech. 2015, 6, 1518-1540. [CrossRef] [PubMed]

5. Thorman, R.M.; Kumar, T.P.R.; Fairbrother, D.H.; Ingolfsson, O. The role of low-energy electrons in focused electron beam induced deposition: Four case studies of representative precursors. Beilstein. J. Nanotech. 2015, 6, 1904-1926. [CrossRef] [PubMed]

6. Gazzadi, G.C.; Frabboni, S.; Menozzi, C. Suspended nanostructures grown by electron beam-induced deposition of Pt and TEOS precursors. Nanotechnology 2007, 18, 445709. [CrossRef]

7. Molhave, K.; Madsen, D.N.; Dohn, S.; Boggild, P. Constructing, connecting and soldering nanostructures by environmental electron beam deposition. Nanotechnology 2004, 15, 1047-1053. [CrossRef]

8. Winkler, R.; Fowlkes, J.D.; Rack, P.D.; Plank, H. 3D nanoprinting via focused electron beams. J. Appl. Phys. 2019, 125. [CrossRef]

9. Bret, T.; Utke, I.; Hoffmann, P.; Abourida, M.; Doppelt, P. Electron range effects in focused electron beam induced deposition of 3D nanostructures. Microelectron. Eng. 2006, 83, 1482-1486. [CrossRef]

10. Kometani, R.; Warisawa, S.; Ishihara, S. The 3D nanostructure growth evaluations by the real-time current monitoring on focused-ion-beam chemical vapor deposition. Microelectron. Eng. 2010, 87, 1044-1048. [CrossRef]

11. Weirich, P.M.; Winhold, M.; Schwalb, C.H.; Huth, M. In situ growth optimization in focused electron-beam induced deposition. Beilstein. J. Nanotech. 2013, 4, 919-926. [CrossRef] [PubMed]

12. Winhold, M.; Weirich, P.M.; Schwalb, C.H.; Huth, M. Modeling the in-situ conductance optimization process in focused electron-beam-induced deposition. Microelectron. Eng. 2014, 121, 42-46. [CrossRef]

13. Fowlkes, J.D.; Winkler, R.; Lewis, B.B.; Stanford, M.G.; Plank, H.; Rack, P.D. Simulation-guided 3D nanomanufacturing via focused electron beam induced deposition. ACS Nano 2016, 10, 6163-6172. [CrossRef] [PubMed]

14. Keller, L.; Al Mamoori, M.K.I.; Pieper, J.; Gspan, C.; Stockem, I.; Schroder, C.; Barth, S.; Winkler, R.; Plank, H.; Pohlit, M.; et al. Direct-write of free-form building blocks for artificial magnetic 3D lattices. Sci. Rep. 2018, 8, 6160. [CrossRef] [PubMed]

15. Utke, I.; Friedli, V.; Michler, J.; Bret, T.; Multone, X.; Hoffmann, P. Density determination of focused-electron-beam-induced deposits with simple cantilever-based method. Appl. Phys. Lett. 2006, 88, 031906. [CrossRef]

16. Randolph, S.J.; Fowlkes, J.D.; Rack, P.D. Effects of heat generation during electron-beam-induced deposition of nanostructures. J. Appl. Phys. 2005, 97, 124312. [CrossRef]

17. Mutunga, E.; Winkler, R.; Sattelkow, J.; Rack, P.D.; Plank, H.; Fowlkes, J.D. Impact of electron-beam heating during 3D nanoprinting. ACS Nano 2019, 13, 5198-5213. [CrossRef] 
18. Skoric, L.; Sans-Hernández, D.; Meng, F.; Donnelly, C.; Merino-Aceituno, S.; Fernandez-Pacheco, A. Layer-by-layer growth of complex-shaped three-dimensional nanostructures with focused electron beams. (accepted).

19. Fowlkes, J.D.; Winkler, R.; Lewis, B.B.; Fernández-Pacheco, A.; Skoric, L.; Sans-Hernández, D.; Stanford, M.G.; Mutunga, E.; Rack, P.D.; Plank, H. High-fidelity 3D-nanoprinting via focused electron beams: Computer-aided design (3BID). ACS Appl. Nano Mater. 2018, 1, 1028-1041. [CrossRef]

20. Winkler, R.; Lewis, B.B.; Fowlkes, J.D.; Rack, P.D.; Plank, H. High-fidelity 3D-nanoprinting via focused electron beams: Growth fundamentals. ACS Appl. Nano Mater. 2018, 1, 1014-1027. [CrossRef]

21. Winkler, R.; Schmidt, F.P.; Haselmann, U.; Fowlkes, J.D.; Lewis, B.B.; Kothleitner, G.; Rack, P.D.; Plank, H. Direct-write 3D nanoprinting of plasmonic structures. ACS Appl. Mater. Int. 2017, 9, 8233-8240. [CrossRef]

22. Höflich, K.; Feichtner, T.; Hansjurgen, E.; Haverkamp, C.; Kollmann, H.; Lienau, C.; Silies, M. Resonant behavior of a single plasmonic helix. Optica 2019, 6, 1098-1105. [CrossRef]

23. Sanz-Hernández, D.; Fernández-Pacheco, A. Modelling focused electron beam induced deposition beyond langmuir adsorption. Beilstein. J. Nanotech. 2017, 8, 2151-2161. [CrossRef] [PubMed]

(C) 2019 by the authors. Licensee MDPI, Basel, Switzerland. This article is an open access article distributed under the terms and conditions of the Creative Commons Attribution (CC BY) license (http://creativecommons.org/licenses/by/4.0/). 